\title{
Allocution du président entrant
}

C'est un grand honneur que d'avoir à assumer la présidence de la Société Française de Physique, société prestigieuse ayant compté nombre de membres illustres. Depuis trois ans, la présidente sortante, Michèle Leduc, le Bureau et le Conseil d'administration de la SFP ont abattu un travail considérable pour la Société, pour améliorer ses structures, diversifier ses actions et renforcer sa présence dans la communauté.

Mon action à la tête de la SFP veut s'inscrire dans la continuité : renforcer son implantation dans la communauté française en poursuivant les campagnes d'adhésion en cours ; améliorer la représentation des domaines scientifiques actifs, en particulier des domaines émergents, en son sein ; accroître la visibilité de la SFP à l'extérieur.

C'est une constatation faite depuis longtemps que la SFP ne rassemble qu'une petite partie de l'ensemble des chercheurs, ingénieurs et enseignants dont l'activité est liée aux sciences physiques, et exerçant en France. Les raisons en sont certainement multiples : culturelles, liées au caractère individualiste et peu associatif prévalant dans l'hexagone ; dispersion de la communauté entre de nombreuses sociétés spécialisées (optique, mécanique, acoustique, etc.) ; faible présence hors du milieu académique... Cette faible représentativité de la SFP limite l'efficacité de ses interventions dans les champs politique, scientifique et éducatif, ainsi qu'auprès du grand public. À titre de comparaison, I'loP et la DPG, les sociétés de physique britannique et allemande, reçoivent régulièrement des missions officielles de la part de leurs gouvernements.

Une campagne de recrutement a été lancée l'an dernier en direction des jeunes scientifiques du milieu académique, avec l'aide du CNRS et du CEA. Elle va se poursuivre en direction des élèves des écoles d'ingénieurs et des étudiants de master dans les universités. Il va falloir dans un premier temps attirer les jeunes scientifiques et ingénieurs, et ensuite les fidéliser en leur fournissant un forum de discussion (mise en place de réseaux, aide à l'emploi, etc.). Une réflexion dans ce sens est menée par un petit groupe autour de Jean-Paul Hermann et Pierre Billaud. Parallèlement, un comité "physique et industrie » sera mis en place pour réfléchir aux moyens d'améliorer la présence des scientifiques du milieu industriel au sein de la SFP et leur apport aux grands débats en cours. L'ouverture de la SFP, et de ses sections locales, au monde industriel est encore à réaliser.

En étroite relation avec les autres disciplines scientifiques, la physique est une science dont les frontières sont en évolution permanente. La SFP doit s'y adapter et devancer ces évolutions. La nécessité de couvrir l'ensemble du champ des sciences physiques, et particulièrement les domaines rapidement émergents aux interfaces, vont mener la SFP à créer de nouvelles divisions transdisciplinaires, différentes des divisions thématiques existantes : on peut penser à des divisions de physique "Sciences de la Vie " et "TerreEnvironnement ». Cette ouverture m'apparaît nécessaire pour que les physiciens travaillant dans ces domaines puissent se retrouver pleinement dans la communauté plus large des sciences physiques. Elle permettra aussi à la SFP d'intervenir avec plus de poids dans les grands débats de société présentant une composante scientifique : énergie, environnement, santé...

Le rayonnement de la SFP à l'extérieur ne peut s'établir de façon durable que si celle-ci est fortement implantée dans sa communauté. À partir de cette base, elle pourra poursuivre et amplifier ses efforts de communication vers le grand public et la société au sens large, dans la lignée de l'Année Mondiale de la Physique en 2005 qui avait été une grande réussite. Il lui faut continuer ces activités de promotion de la physique et des sciences physiques, s'impliquer fortement dans les actions en cours (50 ans du Laser cette année) et lancer de nouvelles idées de célébrations scientifiques nationales et internationales.

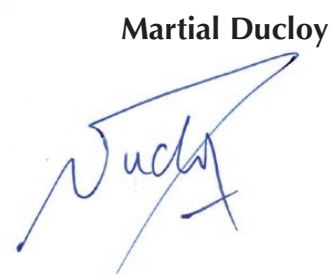

